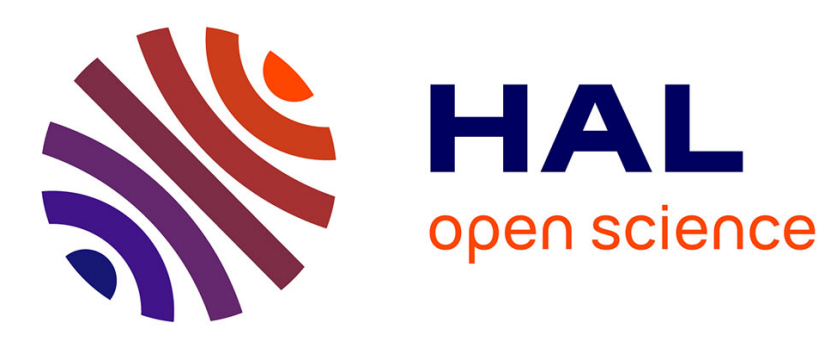

\title{
Mathematical and Numerical Analysis of a Class of Non-linear Elliptic Equations in the Two Dimensional Case
}

\author{
Noureddine Alaa, Jean Rodolphe Roche, A. Cheggour
}

\section{- To cite this version:}

Noureddine Alaa, Jean Rodolphe Roche, A. Cheggour. Mathematical and Numerical Analysis of a Class of Non-linear Elliptic Equations in the Two Dimensional Case. Jul 2006, pp.926-936. hal00095264

\section{HAL Id: hal-00095264 \\ https://hal.science/hal-00095264}

Submitted on 15 Sep 2006

HAL is a multi-disciplinary open access archive for the deposit and dissemination of scientific research documents, whether they are published or not. The documents may come from teaching and research institutions in France or abroad, or from public or private research centers.
L'archive ouverte pluridisciplinaire HAL, est destinée au dépôt et à la diffusion de documents scientifiques de niveau recherche, publiés ou non, émanant des établissements d'enseignement et de recherche français ou étrangers, des laboratoires publics ou privés. 


\title{
Mathematical and Numerical Analysis of a Class of Non-linear Elliptic Equations in the Two Dimensional Case.
}

\author{
Nour Eddine Alaa ${ }^{1}$, Abderrahim Cheggour ${ }^{1}$ and Jean R. Roche ${ }^{2}$ \\ 1 Département de Mathématiques et Informatique, Université des Sciences et \\ Techniques Cadi Ayyad, B.P. 618, Guéliz, Marrakech, Maroc \\ alaa@fstg-marrakech.ac.ma \\ 2 I.E.C.N., Université Henri Poincaré, B.P. 239, 54506 Vandoeuvre lès Nancy, \\ France roche@iecn.u-nancy.fr
}

Summary. The aim of this paper is to show the existence and present a numerical analysis of weak solutions for a quasi-linear elliptic problem with Dirichlet boundary conditions in a domain $\Omega$ and data belonging to $L^{1}(\Omega)$. A numerical algorithm to compute a numerical approximation of the weak solution is described and analyzed. Numerical examples are presented and commented.

\section{Introduction}

The principal objective of this work is to give a result of existence and present a numerical analysis of weak solutions for the following quasi-linear elliptic problem:

$$
\left\{\begin{array}{l}
-\Delta u(x)+G(x, \nabla u(x))=F(x, u(x))+f(x) \text { in } \Omega \\
u(x)=0 \text { on } \partial \Omega
\end{array}\right.
$$

where $G, F$ are Caratheodory non negative functions. The function $f \in L^{1}(\Omega)$ is given finite non negative. The domain $\Omega \subset \mathbb{R}^{N}$ is open and bounded. Such problems arise from biological, chemical and physical systems.

The two essential ingredients to the analysis of this problem are the convexity of $s \rightarrow G(x, s)$ and that $G(x, s)$ is sub-quadratic w.r.t. $s$ namely:

$$
G(x, s) \leq C\left(k(x)+\|s\|^{2}\right), \quad \text { where } \quad k(x) \in L^{1}(\Omega) \text { and } C>0 .
$$

Then the problem (1) has a solution in $W_{0}^{1, q}(\Omega)$ where $1 \leq q<N /(N-1)$, $N \geq 2$, provided that (1) has a super-solution in $W_{0}^{1,1}(\Omega)$.

In previous work [4] the authors show the existence of a weak solution in the one-dimensional case and with arbitrary growth of the non linearity and data measure. 
We study a numerical method to compute the solution of the problem (1). In the first step we compute a super solution using a domain decomposition method. In the second step we compute a sequence of solutions of an intermediate problem obtained by using the Yosida approximation of $G$. This sequence converges to the weak solution of the problem (1).

\section{Statement of the Main Result}

Throughout this paper we suppose

$$
f \in L^{1}(\Omega), f \geq 0 .
$$

The functions $G: \Omega \times \mathbb{R}^{N} \rightarrow[0,+\infty[$ and $F: \Omega \times \mathbb{R} \rightarrow[0,+\infty[$ are such that:

$G, F$ are measurable, $r \rightarrow G(x, r)$ and $u \rightarrow F(x, u)$ are continuous.

$G$ is convex in $r$ and $F$ is nondecreasing in $u$,

$$
\begin{gathered}
G(x, 0)=\min \left\{G(x, r), r \in \mathbb{R}^{N}\right\}=0, \text { and } F(x, 0)=0, \\
G(x, r) \leq C\left(|r|^{2}+K(x)\right), \\
F(x, u) \in L^{1}(\Omega) \text { for every } u \in \mathbb{R}
\end{gathered}
$$

with a constant $C>0$ and $K \in L^{1}(\Omega)$.

We introduce now the notion of weak solutions of problem (1).

Definition 1. A function $u$ is said to be a weak solution of the problem (1), if

$$
\left\{\begin{array}{l}
u \in W_{0}^{1,1}(\Omega), G(x, \nabla u) \text { and } F(x, u) \in L^{1}(\Omega) \\
-\Delta u+G(x, \nabla u)=F(x, u)+f \text { in } \mathcal{D}^{\prime}(\Omega)
\end{array}\right.
$$

We will be interested in proving the existence of weak positive solutions of problem (1).

Theorem 1. Under hypotheses (3)-(7), and assuming that there exists $w$ such that

$$
\left\{\begin{array}{l}
w \in W_{0}^{1,1}(\Omega), \quad F(x, w) \in L^{1}(\Omega), \\
-\Delta w=F(x, w)+f \quad \text { in } \mathcal{D}^{\prime}(\Omega),
\end{array}\right.
$$

the problem (1) has a positive weak solution. 


\section{Proof of Theorem 1}

\subsection{Approximation Scheme}

We consider the sequence defined by $u_{0}=w$ and for $n \geq 0, u_{n+1}$ is the solution of the problem

$$
\left\{\begin{array}{l}
-\Delta u_{n+1}+G_{n+1}\left(x, \nabla u_{n+1}\right)=F\left(x, u_{n}\right)+f \text { in } \mathcal{D}^{\prime}(\Omega), \\
u_{n+1} \in W_{0}^{1,1}(\Omega), G_{n+1}\left(x, \nabla u_{n+1}\right) \in L^{1}(\Omega)
\end{array}\right.
$$

where $G_{n}(x, r)$ denotes the Yosida approximation of $G(x, r)$. The function $G_{n}(x, r)$ is convex in $r$, increases pointwise to $G(x, r)$ as $n$ tends to $\infty$ and satisfies

$$
G_{n} \leq G_{n+1} \leq G, \quad\left\|G_{n, r}(x, r)\right\|_{\infty} \leq n
$$

where $G_{n, r}$ denotes a section of subdifferential of $G_{n}$ with respect to $r$.

The classical works ([3], [6], [9]) combined with an induction argument can be applied to prove that (11) has a solution such that

$$
0 \leq u_{n+1} \leq u_{n} \leq w
$$

\subsection{Estimates and Convergence}

Let $\left\{u_{n}\right\}_{n}$ be a sequence defined as above. By integrating (11) in $\Omega$ and using (13) we obtain

$$
\int_{\Omega} G_{n+1}\left(x, \nabla u_{n+1}\right) d x \leq \int_{\Omega} F(x, w) d x+\int_{\Omega} f(x) d x .
$$

Therefore $\left\|\Delta u_{n+1}\right\|_{L^{1}(\Omega)}$ is bounded. Then there exists a subsequence still denoted by $u_{n}$ for simplicity, such that $u_{n}$ converges strongly to some $u$ in $W_{0}^{1, q}(\Omega), 1 \leq q<N /(N-1)$, and $\left(u_{n}, \nabla u_{n}\right)$ converges to $(u, \nabla u)$ almost everywhere in $\Omega$ (see [8]).

Let us prove that $u$ is in fact a solution of problem (1). According to the definition 2.1 , we only have to show that

$$
-\Delta u+G(x, \nabla u)=F(x, u)+f \text { in } \mathcal{D}^{\prime}(\Omega) .
$$

We know that $F\left(x, u_{n}\right) \longrightarrow F(x, u)$ strongly in $L^{1}(\Omega)$ and, for almost every $x$ in $\Omega$, there holds $G_{n+1}\left(x, \nabla u_{n+1}(x)\right) \longrightarrow G(x, \nabla u(x))$.

Then there exists a non-negative measure $\mu$ (see [7]) such that

$-\Delta u_{n}+G_{n+1}\left(\nabla u_{n+1}\right)-F\left(u_{n}\right)-f \longrightarrow-\Delta u+G(\nabla u)-F(u)-f+\mu$ in $\mathcal{D}^{\prime}(\Omega)$, as $n$ goes to $\infty$.

On the other hand

$$
-\Delta u_{n+1}+G_{n+1}\left(x, \nabla u_{n+1}\right)=F\left(x, u_{n}\right)+f \longrightarrow F(x, u)+f \text { in } L^{1}(\Omega) .
$$


Consequently

$$
-\Delta u+G(x, \nabla u) \leq F(x, u)+f \text { in } \mathcal{D}^{\prime}(\Omega) .
$$

Therefore to conclude the proof of theorem 1, we must establish the opposite inequality. To this end we introduce the following test function

$$
\psi \exp \left(-C u_{n+1}\right) H\left(\frac{u_{n+1}}{k}\right)
$$

where $H \in C^{1}(\mathbb{R}), 0 \leq H(s) \leq 1, H(s)=0$ if $|s| \geq 1$ and $H(s)=1$ if $|s| \leq$ $\frac{1}{2}, C$ is given by relation (7) and $\psi \leq 0, \psi \in H_{0}^{1}(\Omega) \cap L^{\infty}(\Omega)$. We multiply the equation satisfied by $u_{n+1}$ in (11) by this test function and we integrate in $\Omega$, to obtain

$$
\int_{\Omega}\left(f_{n}+F\left(x, u_{n}\right)\right) \psi \exp \left(-C u_{n+1}\right) H\left(\frac{u_{n+1}}{k}\right) d x=I_{1}+I_{2}+I_{3},
$$

where

$$
\begin{aligned}
& I_{1}=\int_{\Omega} \nabla u_{n+1} \nabla \psi \exp \left(-C u_{n+1}\right) H\left(\frac{u_{n+1}}{k}\right) d x, \\
& I_{2}=\frac{1}{k} \int_{\Omega}\left|\nabla u_{n+1}\right|^{2} \psi \exp \left(-C u_{n+1}\right) H^{\prime}\left(\frac{u_{n+1}}{k}\right) d x, \\
& I_{3}=\int_{\Omega}\left(G_{n+1}\left(x, \nabla u_{n+1}\right)-C\left|\nabla u_{n+1}\right|^{2}\right) \psi \exp \left(-C u_{n+1}\right) H\left(\frac{u_{n+1}}{k}\right) d x .
\end{aligned}
$$

By investigating separately each term, we get

$$
\begin{aligned}
\lim _{n \rightarrow \infty} I_{1} & =\int_{\Omega} \nabla u \nabla \psi \exp (-C u) H\left(\frac{u}{k}\right) d x \\
\text { and } \lim _{k \rightarrow \infty} I_{2} & =0 \text { uniformly on } n .
\end{aligned}
$$

Now we investigate the remaining term $I_{3}$. Since $G_{n+1}$ satisfies the inequality (7), $\psi \leq 0$, and by applying Fatou's lemma, we obtain

$$
\lim _{n \rightarrow \infty} I_{3} \geq \int_{\Omega}\left(G(x, \nabla u)-C|\nabla u|^{2}\right) \psi \exp (-C u) H\left(\frac{u}{k}\right) d x .
$$

Finally we have shown

$$
\begin{aligned}
& \int_{\Omega} \nabla u \nabla \psi \exp (-C u) H\left(\frac{u}{k}\right) d x+\int_{\Omega} \psi\left(G(x, \nabla u)-C|\nabla u|^{2}\right) \exp (-C u) H\left(\frac{u}{k}\right) d x \\
& +\omega\left(\frac{1}{k}\right) \leq \int_{\Omega}(F(x, u)+f) \psi \exp (-C u) H\left(\frac{u}{k}\right) d x,
\end{aligned}
$$

where $\omega(\varepsilon)$ denotes a quantity that tends to 0 when $\varepsilon$ tends to 0 . Now we choose $\psi=-\varphi \exp (C u) H\left(\frac{u}{k}\right)$, where $\varphi \geq 0, \varphi \in \mathcal{D}(\Omega)$ and we replace $\psi$ by 
this value in the previous inequality to get after appropriate calculations and using that the third term is equivalent to $\omega\left(\frac{1}{k}\right)$

$$
\begin{aligned}
& -\int_{\Omega} \nabla u \nabla \varphi H\left(\frac{u}{k}\right)^{2} d x-\int_{\Omega} \varphi G(x, \nabla u) H\left(\frac{u}{k}\right)^{2} d x+\omega\left(\frac{1}{k}\right) \\
& \leq-\int_{\Omega}(F(x, u)+f) \varphi H\left(\frac{u}{k}\right)^{2} d x .
\end{aligned}
$$

We finally pass to the limit as $k$ tends to infinity and we use the fact that $\lim _{k \rightarrow \infty} H\left(\frac{u}{k}\right)=1$, to conclude for every $\varphi \geq 0, \varphi \in \mathcal{D}(\Omega)$ that

$$
\int_{\Omega} \nabla u \nabla \varphi d x+\int_{\Omega} \varphi G(x, \nabla u) d x \geq \int_{\Omega}(F(x, u)+f) \varphi d x .
$$

This finishes the proof of the theorem 1 .

\section{Numerical Method}

\subsection{Introduction}

In this section we present the numerical method to solve the equation (1) in $\mathbb{R}^{2}$. Formally the algorithm can be formulated in the following way:

1) Find $\bar{w} \in H_{0}^{1}(\Omega)$ such that:

$$
-\Delta \bar{w}(x) \geq F(x, \bar{w})+f \text { in } \Omega .
$$

2) Given $u_{0}=\bar{w}$ we compute a sequence, $\left\{u_{n}\right\}_{n}$, solution in $H_{0}^{1}(\Omega)$ of the non linear equation:

$$
-\Delta u_{n+1}(x)+G_{n+1}\left(x, \nabla u_{n+1}\right)=F\left(x, u_{n}\right)+f \text { in } \Omega .
$$

Both problems (21) and (22) are non-linear, and if (21) has a solution, in theorem 1 we have shown that (22) then also has a solution.

\subsection{Numerical Algorithm}

This subsection summarizes the algorithm introduced in the previous subsection.

1) First step: given $\bar{w}^{0}=0$, iteratively for $k=1$ until convergence we compute $\bar{w}^{k+1}=\bar{w}^{k}+\delta$ where at each iteration $\delta$ is the solution of the linear problem:

$$
\left\{\begin{array}{l}
-\Delta \delta(x)-\frac{\partial F\left(x, \bar{w}^{k}\right)}{\partial r} \delta(x)=\Delta \bar{w}^{k}(x)+F\left(x, \bar{w}^{k}\right)+f \text { in } \Omega, \\
\delta(x)=0 \text { on } \partial \Omega .
\end{array}\right.
$$

To solve at each iteration the linear problem (23) we consider the domain decomposition method which will be introduced as follows: 
a) We compute $c_{\infty}=\left\|\frac{\partial F\left(\bar{w}^{k}\right)}{\partial r}\right\|_{\infty}$. Determine an overlapping subdomain decomposition $\Omega_{i}, i=1, \ldots, m$ such that $\Omega=\cup_{i=1}^{m} \Omega_{i}$ and satisfies:

$$
\max \left\{\operatorname{mes}\left(\Omega_{i}\right), i=1, \ldots, m\right\}<\min \left(\frac{c_{0} \pi^{2}}{c_{\infty}}, \frac{\pi}{2 \sqrt{c_{\infty}}}\right) .
$$

We denote by $m$ the number of subdomains $\Omega_{i}$ and $\partial \Omega_{i}$ is the boundary of $\Omega_{i}$.

b) Iteratively:

for $l=1$, ..until convergence and for $i=1, \ldots, m$ we solve the following subdomain problems:

$$
\left\{\begin{array}{l}
-\Delta \delta_{i}^{l}(x)-\frac{\partial F\left(x, \bar{w}^{k}\right)}{\partial r} \delta_{i}^{l}(x)=\Delta \bar{w}^{k}(x)+F\left(x, \bar{w}^{k}\right)+f \text { in } \Omega_{i}, \\
\delta_{i}^{l}(x)=\delta_{j}^{l-1}(x), \text { on } \partial \Omega_{i} \cap \Omega_{j} \\
\delta_{i}^{l}(x)=0, \text { on } \partial \Omega \cap \partial \Omega_{i}
\end{array}\right.
$$

On each subdomain $\Omega_{i}$ we consider a finite element approximation method with $N_{i}$ elements. At the end of the $l$-th loop we have computed an approximate discrete solution of the linear indefinite problem (23).

2) At this step for $u_{0}=\bar{w}$, iteratively for $n=1$, until convergence we solve the following non-linear problem

$$
\left\{\begin{array}{c}
-\Delta u_{n}(x)+G_{n}\left(x, \nabla u_{n}\right)=F\left(x, u_{n-1}\right)+f \text { in } \Omega, \\
u_{n}(x)=0 \text { on } \partial \Omega .
\end{array}\right.
$$

At each $n$-th step the problem (26) is solved by using a Newton method. The discrete approximation of the solution of (1) is obtained at the end of the $n$-th loop.

\subsection{Convergence of the Domain Decomposition Method}

To simplify, without lost of generality, we assume that we can consider a two-domain decomposition $\Omega=\Omega_{1} \bigcup \Omega_{2}$ such that:

$$
\max \left\{\operatorname{mes}\left(\Omega_{i}\right), i=1,2\right\}<\min \left(\frac{c_{0} \pi^{2}}{c_{\infty}}, \frac{\pi}{2 \sqrt{c_{\infty}}}\right) .
$$

Now to prove the convergence of the Schwarz overlapping domain decomposition algorithm applied to problem (23), we consider two problems:

$$
\left\{\begin{array}{c}
-\Delta v_{1}(x)+c(x) v_{1}(x)=h(x) \text { in } \Omega_{1} \\
v_{1}(x)=0 \text { on } \partial \Omega \cap \partial \Omega_{1} ; v_{1}(x)=v_{2}(x) \text { on } \partial \Omega_{1} \cap \Omega_{2}
\end{array}\right.
$$

and

$$
\left\{\begin{array}{l}
-\Delta v_{2}(x)+c(x) v_{2}(x)=h(x) \text { in } \Omega_{2}, \\
v_{2}(x)=v_{1}(x) \text { on } \partial \Omega_{2} \cap \Omega_{1} ; v_{2}(x)=0 \text { on } \partial \Omega \cap \Omega_{2} .
\end{array}\right.
$$


Let $v$ be

$$
v= \begin{cases}v_{1} & \text { in } \Omega_{1}, \\ v_{2} & \text { in } \Omega_{2}\end{cases}
$$

$v_{1}=v_{2}$ in $\Omega_{1} \cap \Omega_{2}$.

With the restriction (27) we can suppose the existence of a solution of (28) in $W_{0}^{1, q}\left(\Omega_{1}\right)$ and a solution of (29) in $W_{0}^{1, q}\left(\Omega_{2}\right)$.

Then, if $v^{0}$ is an initialization function defined in $\Omega$ and vanishing in $\partial \Omega$, we define for $k \geq 0$ two sequences $v_{i}^{k}, i=1,2$ solving the following problems:

$$
\left\{\begin{array}{c}
-\Delta v_{1}^{k+1}(x)+c(x) v_{1}^{k+1}(x)=h(x) \quad \text { in } \Omega_{1}, \\
v_{1}^{k+1}(x)=0 \text { on } \partial \Omega \cap \partial \Omega_{1} ; v_{1}^{k+1}(x)=v_{2}^{k}(x) \text { on } \partial \Omega_{1} \cap \Omega_{2}
\end{array}\right.
$$

and

$$
\left\{\begin{array}{c}
-\Delta v_{2}^{k+1}(x)+c(x) v_{2}^{k+1}(x)=h(x) \quad \text { in } \Omega_{2}, \\
v_{2}^{k+1}(x)=v_{1}^{k}(x) \text { on } \partial \Omega_{2} \cap \Omega_{1} ; v_{2}^{k+1}(x)=0 \text { on } \partial \Omega \cap \Omega_{2} .
\end{array}\right.
$$

Theorem 2. Assume $\Omega_{1}$ and $\Omega_{2}$ with the restriction (27). Then the sequence $v^{k}$ converges to $v$ in $W_{0}^{1, q}\left(\Omega_{1}\right)$ and $W_{0}^{1, q}\left(\Omega_{2}\right)$.

Proof. We give here an idea of the proof.

Let $d^{k}=v_{1}^{k}-v$ in $\Omega_{1}$ and $e^{k}=v_{2}^{k}-v$ in $\Omega_{2}$ then $d^{k} \in L^{\infty}\left(\Omega_{1}\right)$ and $e^{k} \in L^{\infty}\left(\Omega_{2}\right)$.

Thanks to the maximum principle we prove the following inequalities:

$$
\left\|d^{k+2}\right\|_{\infty} \leq \gamma\left\|d^{k}\right\|_{\infty} \text { and }\left\|e^{k+2}\right\|_{\infty} \leq \gamma\left\|e^{k}\right\|_{\infty}
$$

where $\gamma<1$.

But to be able to apply the maximum principle it will be necessary that the subdomains $\Omega_{1}$ and $\Omega_{2}$ verify the restriction (27).

\subsection{Numerical Results}

The algorithm introduced in the previous section has been implemented numerically for the model problem (1) where:

$G(x, r)=|r|^{p}=\left(r_{1}^{2}+r_{2}^{2}\right)^{\frac{p}{2}}$ and $r=\left(r_{1}, r_{2}\right) \in \mathbb{R}^{2}$ for $1<p<\infty$.

$F(x, s)=\eta s^{q}$ where $s \in \mathbb{R}^{+}$and $1<q<\infty$.

$f(x)=x_{1}^{\alpha}+x_{2}^{\beta}$ where $x=\left(x_{1}, x_{2}\right) \in \Omega$ and $-1<\alpha, \beta<\infty$.

The number of subdomains is not fixed, it changes at each iteration according to the criterion (27). In figure 1 we can see the solution shape when the algorithm converges with $m=36$ subdomains.

To study the convergence history of the numerical simulation plotted in figure 1 we consider two steps. In the first step, where we compute a supersolution, we observe the evolution of the number of subdomains: it goes from $m=4$ subdomains to $m=36$ subdomains in seven iterations according to criterion (27). Simulation stops after 34 iterations when the residual is less 


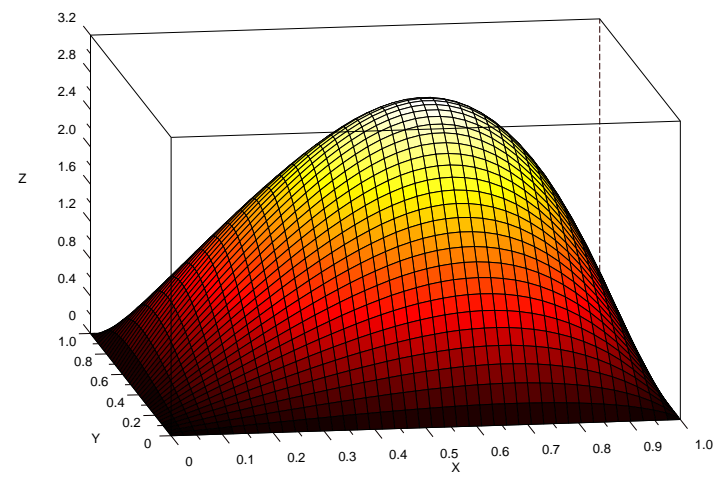

Fig. 1. $\eta=45, p=q=3, \alpha=\beta=2, m=36$

than $10^{-6}$. In the second step, starting with the super-solution computed in the previous step we perform nine iterations of the Yosida approximation described in section 3 and the simulation stops when the correction computed is in uniform norm less than $10^{-6}$.

\section{References}

1. Alaa, N., Maach, F., Mounir, I.: Existence for some quasilinear elliptic systems with critical growth nonlinearity and $L^{1}$ data. Journal of Applied Analysis, 11, 81-94 (2005)

2. Alaa, N., Mounir, I.: Global existence for reaction-diffusion systems with mass control and critical growth with respect to the gradient. Journal of Mathematical Analysis and Applications, 253, 532-557 (2001)

3. Alaa, N., Pierre, M.: Weak solution of some quasilinear elliptic equations with measures. SIAM J. Math.Anal, 24, 23-35 (1993)

4. Alaa, N., Roche, J.R.: Theoretical and numerical analysis of a class of nonlinear elliptic equations. Mediterr. J. Math, 2, 327-344 (2005)

5. Amann, H., Crandall, M.G.: On some existence theorems for semi linear equations. Indiana Univ. Math. J, 7, 779-790 (1978)

6. Boccardo, L., Murat, F., Puel, J.P.: Existence de solutions non bornées pour certaines équations quasi-linéaires. Portugaliae Math., 41, 507-534 (1982)

7. Brezis, H.: Analyse fonctionnelle appliquée, Masson, Paris (1980)

8. Brezis, H., W. Strauss, W.: Semilinear elliptic equation in $L^{1}$. J. Math. Soc. Japan, 25, 565-590 (1973)

9. Lions, P.L.: Résolution de problèmes elliptiques quasilinéaires. Arch. Ration. Mech. Analysis, 74, 335-353 (1980)

10. Porretta, A.: Existence for elliptic equations in $L^{1}$ having lower order terms with natural growth. Portugaliae Math., 57, 179-190 (2000) 